\title{
BMJ Open Protecting older people living in care homes from COVID-19: a protocol for a mixed-methods study to understand the challenges and solutions to implementing social distancing and isolation
}

\author{
Joanne M Fitzpatrick (D) ," Anne Marie Rafferty (D) , ${ }^{1}$ Shereen Hussein (D) , \\ Ivanka Ezhova (10 , ${ }^{1}$ Sinead Palmer (1) , ${ }^{3}$ Richard Adams, ${ }^{4}$ Lindsay Rees, ${ }^{5}$ \\ Sally Brearley (D) , ${ }^{6}$ Sarah Sims (D) , ${ }^{1}$ Ruth Harris (D) ${ }^{1}$
}

To cite: Fitzpatrick JM, Rafferty AM, Hussein S, et al. Protecting older people living in care homes from COVID-19: a protocol for a mixed-methods study to understand the challenges and solutions to implementing social distancing and isolation. BMJ Open 2021;11:e050706. doi:10.1136/ bmjopen-2021-050706

- Prepublication history for this paper is available online. To view these files, please visit the journal online (http://dx.doi. org/10.1136/bmjopen-2021 050706).

Received 26 February 2021 Accepted 16 June 2021

Check for updates

(c) Author(s) (or their employer(s)) 2021. Re-use permitted under CC BY-NC. No commercial re-use. See rights and permissions. Published by BMJ.

For numbered affiliations see end of article.

Correspondence to Joanne M Fitzpatrick; joanne.fitzpatrick@kcl.ac.uk

\section{ABSTRACT}

Introduction Older people living in residential and nursing care homes often have complex needs and are at high risk of poor health outcomes and mortality, especially if they contract COVID-19. Care homes use infection prevention and control measures such as social distancing and isolating residents to protect them from COVID-19. The care home sector has stated that implementing social distancing and isolation when caring for residents is a significant challenge. This paper presents the protocol of a mixed-methods study to explore and understand the real-life experiences of implementing social distancing and isolation of residents in care homes for older people from the perspective of residents, families/friends and staff working in and with care homes. The study aims to develop a toolkit of resources for health and care delivery now and for future outbreaks of infectious diseases. Methods and analysis The study will be conducted in three phases. Phase 1 is a rapid review of evidence to collate knowledge on the mechanisms and measures used by care homes and long-term facilities to socially distance and isolate older people or control the spread of other infectious and contagious diseases. The review results will contribute to participant interviews in phase 2 and toolkit development in phase 3 . Phase 2 will involve case studies with six care homes in England, involving the conduct of individual interviews with residents, families and friends, and staff, collection of care home policies and protocols related to social distancing and isolation for residents, and routinely collected care home data. A focus group with a purposive sample of external key informants will also be conducted. Phase 3 , synthesising findings from phases 1 and 2, will inform the codesign of a toolkit of resources for residents, families/friends and care homes.

Ethics and dissemination The study has been approved by Coventry and Warwick Research Ethics Committee (20/WM/0318). To maximise impact, we will work closely with the Study Committees and the Patient and Public Involvement group to ensure the findings reach key stakeholders, including residents, families/friends, care
Strengths and limitations of this study

- This mixed-methods study will identify the real-life challenges and consequences of providing safe care incorporating social distancing and isolation measures within a care home setting while balancing the potentially significant impacts for residents' psychological, social and physical well-being.

- It will identify novel solutions used by care homes to implement social distancing and isolation for residents.

- It will culminate in a coproduced toolkit that comprises evidence-informed guidance and resources to support care homes, staff, residents and families/ friends during this and possible future pandemics.

- It has been designed and will be conducted with the involvement of experienced service users.

- A study limitation is that restrictions owing to COVID-19 prohibit us from immersing ourselves as researcher-observers in care homes to gain insights into contexts and cultures and observe first-hand activities, interactions and communications.

homes, commissioners and organisations representing care home providers. We will disseminate the study outputs in peer-reviewed and professional journals, at professional conferences and via other knowledge transfer activities with the care home sector, and resident and carer organisations. The toolkit comprising evidenceinformed guidance and resources and a mosaic film will be hosted on a project webpage.

Registration details This project is funded by the National Institute for Health Research (NIHR) Health Services and Delivery Research Programme (Project reference NIHR132541). The views expressed are those of the authors and not necessarily those of the NIHR or the Department of Health and Social Care.

PROSPERO registration number CRD42021226734. 


\section{INTRODUCTION}

During the current COVID-19 pandemic, and for any further surges, it is paramount that older people's care home services remain safe, effective, caring, responsive to individual needs and are well-led. ${ }^{1}$ The sector must be supported to care well for older people and their families and friends and to care well for its workforce. Evidence shows that the care home sector was overlooked in the initial planning of how to contain COVID-19. ${ }^{2}$ The COVID-19 pandemic has had a significant impact on the sector, with care homes experiencing high numbers of COVID-related deaths. ${ }^{3}$ Globally, $41 \%$ of all COVID-19 deaths, for 22 participating countries, were care home residents. ${ }^{4}$ Since the start of the pandemic, 325000 care home residents in these 22 countries have died due to COVID-19, representing one in 20 UK care home residents. ${ }^{4}$ For England, between 20 March 2020 and 15 January 2021, there were 29381 care home resident deaths, accounting for $33 \%$ of all COVID-19 related deaths in England. ${ }^{4}$ In the UK and internationally, care homes caring for older people have faced significant challenges during the pandemic. ${ }^{5-8}$ These challenges include inadequate support to manage infection control effectively. For example, multiple versions of guidance with rapid changes; decision-making at speed to care safely for residents, families/friends and staff; sourcing and funding challenges of personal protective equipment; concerns about testing (who to test, when and how often) and guidance related to the discharge of older people from hospitals to care homes. ${ }^{9} 10$

The care home sector provides care for a vulnerable population of older people, many of whom live with complex and often multiple health needs. ${ }^{11}{ }^{12}$ Those living with complex medical conditions are at high risk of poor health outcomes and mortality if they contract COVID-19. ${ }^{13}$ The risk of older people living in care homes contracting COVID-19 is compounded further by high density and difficulties in maintaining precautions for residents with different needs. For example, implementing social distancing and isolation for those living with dementia who may 'walk with purpose, often called wandering', adding further complexity to safeguarding practices and managing risk. ${ }^{13}$ We use the terms 'social distancing' and 'isolation' as set out in the UK Government document Admission and care of residents in a care home during COVID-19. ${ }^{14}$ This guidance states that care homes 'should be stringent in following social distancing measures for everyone in the care home and supporting those in clinically extremely vulnerable groups to follow shielding guidance' (p 23).

The evidence base lacks support for the delivery of social distancing and isolation as part of infection prevention and control strategies for older people living in care homes. Research is needed to explore and understand the challenges experienced by care homes endeavouring to implement these measures in a way that does not make the home feel like institutional confinement. It is critical to capture the expert ways in which good care homes manage to implement social distancing and isolation requirements in this challenging context and mitigate negative consequences for residents, their families/friends and staff. For residents, potential negative psychosocial and physical repercussions include loneliness, social isolation, low mood, loss of cognitive function, ${ }^{15}$ loss of physical function ${ }^{13}$ and for those living with dementia, a worsening of both cognitive and psychological symptoms. ${ }^{16}$ Possible adverse consequences for families/friends include loss and grief ${ }^{17}$ and care home staff, moral distress, fear and fatigue. ${ }^{101819}$ Our study will complement research that has commenced in the field, for example, family-focused research by Hinsliff-Smith and Brown $^{20}$ to understand the experience of family carers keeping in contact with families and friends living in care homes during the pandemic, and workforce research to examine the impact of COVID-19 on health and care staff working across the UK. ${ }^{1021}$

Our research questions are as follows: what are the challenges, consequences and solutions to implementing social distancing and isolation for older people with different needs living in care homes to protect them from COVID-19? and how can this be achieved safely and in a way that continues to recognise the care home as a home for the older person? This is a 12-month study which began in November 2020.

\section{Aim and objectives}

This study aims to explore and understand the real-life experiences of social distancing and isolation in care homes for older people from the perspective of multiple stakeholders, and to develop a toolkit of evidenceinformed guidance and resources for health and care delivery now and for further outbreaks. The objectives are to:

1. Investigate the mechanisms and measures used by care homes and long-term facilities to socially distance and isolate older people or control the spread of other infectious and contagious diseases, for example, other acute respiratory infections, clostridium difficile and methicillin-resistant staphylococcus aureus.

2. Examine the experiences of residents and families/ friends while residents are social distancing and isolating, how these measures impact on the well-being of residents and families/friends, what is the current practice including innovation in supporting residents and families/friends, and how they are adapting to change.

3. Explore how registered nurses and care staff adapt and manage the delivery of personal, social and psychological care for residents with different needs while maintaining social distancing between staff and residents, residents and other residents, and between staff members.

4. Identify how care home managers, owners and external stakeholders develop, manage and adapt policies, procedures and protocols to implement social distancing and isolation, including workforce organisation, 
training and support, use of communal spaces, visiting and working with external health and social care professionals.

5. Use the findings to develop a toolkit of evidenceinformed guidance and resources detailing which interventions and strategies for social distancing and isolation for residents work well and which do not work in specific situations and contexts to support decision-making about health and care delivery in care homes and to facilitate resilience-building for future planning.

\section{METHODOLOGY \\ Study design}

A mixed-methods study will be conducted in three phases; a rapid evidence review; six in-depth case studies and a multimedia toolkit development that includes a film to narrate the stories of residents, their families/ friends and care home staff. Our decision-making about the research design and methods of data collection has been influenced by the known challenges of researching care homes, ${ }^{22} 23$ including the time-intensive nature of recruiting care homes, care home staff, older people and family members as research participants. ${ }^{24}$ Compounding these challenges is the need for the research team to recruit care homes and participants and to conduct fieldwork remotely due to the current restrictions owing to COVID-19. These measures prohibit us from immersing ourselves as researcher-observers to gain an insight into contexts and cultures and to observe first-hand activities, interactions and communications related to providing social distancing and isolation. Nevertheless, the proposed approach and methods will provide detailed and meaningful accounts of the lived experience and impact of these measures.

\section{Phase 1: Reviewing the evidence (study objective 1)}

A rapid review of evidence will be conducted to identify and assess the strategies previously and currently used by care homes to prevent and control the transmission of COVID-19 and other infectious and contagious diseases. The review results will contribute to the development of the interview guides for phase 2 and the development of the toolkit of guidance and resources in phase 3. The review will be guided by the Cochrane rapid reviews methods group 'evidence-informed guidance for rapid review guidelines'. ${ }^{25}$

Specific review questions are:

1. What mechanisms and measures have been used to implement social distancing and isolation for residents and staff?

2. How are they implemented? What are the challenges and facilitators to implementation?

3. What is the impact of the implemented measures and mechanisms?

a. What are the psychosocial and physical consequences for older people? b. What are the consequences for family members, significant others, staff and organisations?

c. What is the evidence of measures and mechanisms that work for different types of care home, different resident needs, and different ways of organising care delivery?

d. What recommendations have been made after implementation of these measures?

The literature will be searched using the databases MEDLINE; Embase; PsycINFO; CINAHL; HMIC; Social Care Online and Web of Science core collection, and using the search strategy: nursing home* OR care home* OR long-term care* OR long term care* OR aged care facilit* OR agedcare facilit* OR residential care home* AND infect* control* OR infect* prevent* OR cohort* OR zon* OR quarantin* OR social distanc* OR prevent* OR isolat* AND acute respiratory infection* OR clostridium difficile* OR diarrhoea OR vomit* OR methicillin-resistant staphylococcus aureus* OR SARS* OR MERS-CoV* OR flu* OR SARS-Cov19 OR SARS-CoV-2 OR COV* OR Corona* (Limiters From 2003, English language only, aged 65 years and over). The Grey literature will be searched using medRxiv, PDQ-Evidence, NICE Evidence search and TRIP. The website LTCCovid19.org will be searched along with the reference lists of eligible articles. Library specialists have contributed to the development of search strategies.

Any empirical study design will be included and also published literature reviews, best practice guidance and expert opinion. The results from searches will be downloaded to EndNote and any duplicates removed. The title and abstract of all results will be screened for suitability using the inclusion and exclusion criteria. Two reviewers will independently screen $30 \%$ of titles and abstracts, and one reviewer will screen the remaining $70 \%$ of titles and abstracts. A second reviewer will screen all excluded abstracts. One reviewer will screen the full texts of potentially relevant manuscripts. A second reviewer will screen excluded full-text articles. Data will be extracted by a single reviewer using a bespoke data extraction tool. The data extraction tool will be piloted by one reviewer and shared with the team for comment. A second reviewer will check the extracted data for completeness and accuracy.

Data items will include: details of the record, a detailed description of the measure(s) used for social distancing and isolation of residents (what it is; who it is for; how it is implemented, factors supporting or hindering implementation), findings, and author recommendations.

A second reviewer will screen all excluded full-text articles. Findings from the included records will be synthesised using tables and a narrative summary. The narrative synthesis will describe what measures and mechanisms are used to implement social distancing and isolation of residents, what factors support or hinder these, what works and does not work and their impacts. Quality assessment of included articles will be assessed using a valid quality 
assessment tool where available specific to the study design(s)/article type and verified by a second reviewer, for example, checklists from the Critical Appraisal Skills Programme (https:// casp-uk.net/casp-tools-checklists/) or the Joanna Briggs Institute https://jbiglobal/ critical-appraisal-tools.

\section{Phase 2: examining experiences, consequences and solutions} (study objectives 2,3,4)

Case studies

We will use a case study approach ${ }^{26}$ to examine how social distancing and isolation of residents are being implemented in care homes. Six care homes in England with a Care Quality Commission (CQC) quality rating of outstanding, good or requires improvement will be recruited. This selection should help achieve a fuller understanding of a range of care homes' experiences about what has worked well and what has not worked well, in what circumstances and why. Care homes will be purposively sampled to recruit equal numbers of care homes with nursing $(n=2)$, care homes without nursing (residential) $(\mathrm{n}=2)$ and care homes registered as providers of residential and nursing care $(n=2)$. In addition to the type of care home and its CQC rating, the six care homes will be sampled to maximise diversity in terms of several characteristics such as size (larger and smaller bed capacity), ownership (eg, voluntary/not-for-profit, private) and incidences of COVID-19 among the resident population. The cohort of care homes will be geographically spread to include North England, the Midlands and South England. Using the proposed sampling approach and collecting multiple sources of evidence will help us to achieve depth, richness and diversity of data to answer the research question. For each of the six case study care homes, the following data will be collected.

\section{Individual interviews}

One-to-one qualitative, semistructured, remote, online interviews will be conducted with purposively selected staff $(n=5)$, residents $(n=3)$ and resident families/friends $(n=3)$ who consent to participate. We have developed processes and resources to support the well-being of residents, families/friends and staff who participate in the study.

The care manager/deputy will be asked to nominate care staff. The inclusion criteria for staff participants are: (i) permanent staff members; (ii) employees of the participating care home during the pandemic. The exclusion criteria are: (i) staff who are not permanent employees. The care manager/deputy will nominate potential resident participants and families/friends participants. Residents will also be invited to nominate a family member/friend who might like to be interviewed. We will purposively sample residents over the age of 65 years to ensure a range of genders, ethnicities, status as residential and nursing and different health and care needs. Recognising that the prevalence of dementia in UK care homes is around $69 \%$ and increasing ${ }^{27}$ and the importance of including people living with dementia in research, ${ }^{28}{ }^{29}$ we intend to recruit residents living with dementia who can consent to participate in the study. Inclusion criteria are that potential resident participants are mentally and physically able to communicate verbally with a researcher in English, and have the mental capacity to consent. In each care home, the manager will be asked to nominate residents who meet the inclusion criteria and who they think may be interested in taking part in the research. The manager/ project champion will introduce the study to potential resident participants using the approved participant information sheet, which is written and produced in a dementia-friendly way. ${ }^{28}$ All potential participants will have the opportunity to ask a researcher questions about the study. Consent will be sought as per requirements of the UK Research Integrity Office's Code of Practice for Research ${ }^{30}$ and the Mental Capacity Act 2005 Code of Practice. ${ }^{31}$ Written consent will be obtained by the manager/project champion. Some residents may not be able to sign due to physical disabilities (eg, inability to grasp the pen). Other residents are happy to give verbal consent but prefer not to sign anything. In these cases, the consent form can be completed directly on the electronic tablet used for the interview. This will not require a signature, instead the name of the participant and witness can be typed in. This can then be returned to the researcher via email. A modification of Dewing's ${ }^{32}$ process consent framework tailored to social care research will also help guide the process. ${ }^{33}$ This includes the monitoring of ongoing consent, as we are aware that capacity can fluctuate, and a participant may not recall a decision made previously. The researcher will revisit consent again immediately prior to the interview. During the conversation between the researcher and resident, should the researcher at any point have any concerns about the older person's ability to consent to participate, they will not proceed. Residents who do not meet the sampling criteria or who are acutely unwell will not be recruited. Where possible, we will recruit family/friends of nominated residents. Should this not be possible, other family members/friends can be nominated. Inclusion criteria are that potential family/friend participants are mentally and physically able to communicate verbally with a researcher in English, and have the mental capacity to consent. Families/friends who do not meet these criteria will not be recruited.

Remote, online individual interviews with staff members, residents and families/friends will be videorecorded and audio recorded with permission. To help maximise the quality of the interview recordings and footage, we are working with a professional provider, KMTV, to record and edit the interviews. We anticipate interviews lasting between 30 and $60 \mathrm{~min}$. The topic guide will be sent to participants in advance of the interview (see figure 1 for anticipated questions). The interview audiorecordings will be transcribed verbatim, managed using NVIVO-12 software and analysed using thematic 
Residents:

- Have there been any restrictions to where they can go in the care home and for whom they can see?

- Have they noticed that they are seeing fewer people?

- Are they spending more time alone and how that feels?

- What is their view about their feeling of well-being?

- What could be done better/differently in the future?

Family/friend participants:

- What have been the restrictions to being able to visit their family member/friend, and how that feels?

- How are they adapting to change?

- What is their view about the impact on their family member/friend?

- What is their view about how the care home has supported residents and their families/friends?

- What is their view about their own feeling of well-being?

- What could be done better/differently in the future?

\section{Care home staff:}

- How has their working day changed?

- What have been the changes to how they provide care for individual residents?

- What are the challenges of implementing social distancing and isolation for residents?

- What, in their view, has worked well and what has not, and what could be done better/differently in the future?

- What, in their view, is the impact of social distancing and isolation on residents, families/friends, and care home staff?

\section{Focus group participants:}

- What have been the challenges for health and care systems working to protect older people living in care homes from COVID-19?

- What are their views about how policies, procedures and protocols to implement social distancing and isolation have been developed, managed and adapted?

- What, in their view, has worked well and what has not, and what could be done better/differently in the future?

- What are their views about the impacts of social distancing and isolation on residents, families/friends, and care home staff?

- What have been the innovations and creative approaches to protecting older people in care homes from COVID-19, whilst hearing the voice of residents and families/friends?

- What are the take-home messages- how can care homes be supported to protect older people from COVID-19 via social distancing and isolation as part of strategies for infection control and prevention?

Figure 1 Key questions to be included in the schedules for residents, families/friends, care home staff and external key informants.

analysis. $^{34}$ Demographic characteristics for all participants will be collected with permission.

We will engage with the care home manager/owner to identify a dedicated senior staff member to be the project champion. The nominated project champion will be briefed and guided by the research team to support the resident interviews, for example, by e-introducing the older person to the researcher and managing the recording device. The project champion will also assist with the recording device for interviews with staff members if staff participants choose to be interviewed onsite. The video-recordings will be used to inform the toolkit development (phase 3).

\section{Collection of local policies and protocols}

Local policies and protocols relevant to managing the social distancing and isolation of residents will be collected from the manager in each care home, for example, for managing new and returning residents, zoning and cohorting of residents, visiting, staff training and education, education for residents and families/friends, support for residents, families/friends, and staff, and testing of residents and staff. These documentary data will be analysed using thematic analysis. ${ }^{34}$

\section{Collating routinely collected care home data}

To understand as fully as possible contextual factors, data will be collected from each care home for the last 6 months and will include: the number of beds; resident occupancy; $\%$ nursing care and $\%$ residential care; staffing data including absence, redeployment, employment of agency and bank staff; COVID-19 infection rates for residents and staff; hospital admission 
rates for residents; the number of older people transferred from hospitals to care homes, testing of residents and staff; the number of residents who have died including those with COVID-19 on their death certificate, and progress with the vaccine programme for residents and staff. The data will be reported by the care home manager with the approval of the operations manager. These data will be analysed using descriptive summary statistics.

\section{Focus group with external key informants}

For the second component of phase 2, we will conduct a focus group with a purposive sample of external key informants $(n=8)$ beyond the case sites. These participants will have macro-level knowledge and experience relevant to the pandemic for the care home sector, for example, local authority providers, clinical commissioning groups, local resilience fora, the regulator, hospital discharge teams and organisations representing care home providers. The focus group will be conducted remotely online and audiorecorded with permission. We anticipate the focus group lasting between 90 and $120 \mathrm{~min}$. The interview audiorecording will be transcribed verbatim, managed using NVIVO-12 software and analysed using thematic analysis. ${ }^{34}$ To identify and understand similarities and differences, initially, data across sources for each case site will be analysed and then across the case sites, using the constant comparative technique. ${ }^{35}$ Strategies to promote quality will be embedded within our data analysis strategy. ${ }^{36}$ Data analysis for the interviews, focus group and care home documents will be undertaken by the research team and supervised by the lead investigator (JMF). A sample of interviews will be analysed independently by two members of the team to ensure consistency and trustworthiness of analysis. The consolidated criteria for reporting qualitative studies will be used for reporting the study. ${ }^{37}$

\section{Phase 3: synthesis of findings and development of the toolkit (study objective 5)}

We will synthesise the findings from phases 1 and 2 using a narrative approach and convene a series of online stakeholder workshops to coproduce a toolkit of guidance and resources. The content of the toolkit will be presented in different ways to maximise accessibility and impact and will include a mosaic film to help narrate the stories of older people, their families/friends and care home staff. A professional provider, KMTV, will edit the video recordings to produce a mosaic film that will be used to illustrate themes identified for residents, families/friends, and staff participants.

\section{Patient and public involvement}

During the early development of the protocol, we had conversations about the research topic with family members of older people living/have lived in care homes. We consulted with a service user and carer research expert group that has considerable experience contributing to research proposals from a patient and public perspective and is facilitated by the Patient and Public Involvement (PPI) lead for the study (SB). This group comprises mostly older people, many of whom have extensive personal experience of health and care services and several are or have been carers. Email communication was used due to restrictions on meetings. We asked the Group for their views about: the importance and relevance of the research topic; understanding of the terms social distancing and isolating of residents; the proposed design and methods, including suggestions about how to maximise recruitment of residents and families/friends and to make the interview process as user-friendly as possible. We also asked for their feedback on the Plain English Summary to ensure that the language is user friendly and understandable.

Those with experience of having a family member/ friend living in care homes have confirmed that the research is important, feasible and is a key priority for the care home sector. This has influenced the research design, for example, emphasising the importance of including the resident perspective. We are actively involving representatives of service users, carers and the public throughout the research via our PPI group comprising 10 members. This will include reviewing all participant-facing paperwork (eg, study flyers, participant information sheets and consent forms, interview guides) and contributing to the development of the evidence-informed guidance and resources toolkit and disseminating study outputs. Our Study Committees and the PPI Group include representatives of older people living in care homes and their families/friends, experts representing the care home sector, care home nursing and infection control and prevention. The knowledge, expertise and perspectives of these service user and public representatives and those with a professional role in health and social care services will help us achieve this study's objectives.

\section{DISCUSSION}

The evidence base to support the delivery of social distancing and isolation for older people living in care homes is lacking. Research is needed to explore and understand the challenges experienced by care homes endeavouring to implement these measures and in a way that does not make a home feel like institutional confinement. It is critical to capture the expert ways in which care homes manage to implement social distancing and isolation requirements in this challenging environment and mitigate adverse consequences.

Our study will contribute to evidence-informed resident care during social distancing and isolation to prevent and reduce the incidence of COVID-19. We hope that the experience of care, involvement in care and feeling of well-being for residents and families/ 
friends will be improved. Care homes, their staff and networks will have access to evidence-informed guidance and resources. This guidance will help fill a significant evidence gap. We hope the culture of care, organisational resilience and well-being of care home staff will be improved as a result. Learning from our research has the potential to help inform the planning and delivery of health and care for this older people population beyond the acute phase of the pandemic. It will provide support and signposting for any further outbreaks of COVID-19 and potentially other infection-related outbreaks.

\section{Author affiliations}

${ }^{1}$ Florence Nightingale Faculty of Nursing, Midwifery and Palliative Care, King's College London, London, UK

${ }^{2}$ Department of Health Services Research and Policy, London School of Hygiene \& Tropical Medicine, London, UK

${ }^{3}$ Personal Social Services Research Unit, University of Kent, Canterbury, UK

${ }^{4}$ Sears Healthcare Ltd, Newbury, UK

${ }^{5}$ Encore Care Homes, Bournemouth, UK

${ }^{6}$ Kingston University and St George's University of London, London, UK

Twitter Anne Marie Rafferty @amraffer, Shereen Hussein @DrShereeHussein and Ruth Harris @RuthHarris

Contributors JMF, RH, AMR and SH conceived the study. JMF drafted the protocol. RH, AMR, SH, IE, RA, LR and SB contributed to reworking and refining the study objectives, design and methodology. JMF drafted the paper and RH, AMR, SH, $\mathrm{IE}, \mathrm{SP}, \mathrm{SB}, \mathrm{RA}, \mathrm{LR}$ and SS have reviewed and provided comments to improve the manuscript. All authors have read and approved the final version.

Funding This project is funded by the National Institute for Health Research (NIHR) Health Services and Delivery Research Programme (Project number 132541). The views expressed are those of the author(s) and not necessarily those of the NIHR or the Department of Health and Social Care.

\section{Competing interests None declared.}

Patient and public involvement Patients and/or the public were involved in the design, or conduct, or reporting or dissemination plans of this research. Refer to the Methods section for further details.

Patient consent for publication Not required.

Provenance and peer review Not commissioned; externally peer reviewed.

Open access This is an open access article distributed in accordance with the Creative Commons Attribution Non Commercial (CC BY-NC 4.0) license, which permits others to distribute, remix, adapt, build upon this work non-commercially, and license their derivative works on different terms, provided the original work is properly cited, appropriate credit is given, any changes made indicated, and the use is non-commercial. See: http://creativecommons.org/licenses/by-nc/4.0/.

\section{ORCID iDs}

Joanne M Fitzpatrick http://orcid.org/0000-0002-6285-180X

Anne Marie Rafferty http://orcid.org/0000-0003-1010-6581

Shereen Hussein http://orcid.org/0000-0002-7946-0717

Ivanka Ezhova http://orcid.org/0000-0001-8360-0658

Sinead Palmer http://orcid.org/0000-0001-8106-3078

Sally Brearley http://orcid.org/0000-0001-7918-7485

Sarah Sims http://orcid.org/0000-0001-7494-9030

Ruth Harris http://orcid.org/0000-0002-4377-5063

\section{REFERENCES}

1 Care Quality Commission. The five key questions we ask [online], 2018. Available: https://www.cqc.org.uk/what-we-do/how-we-doour-job/five-key-questions-we-ask

2 Sky News. COVID-19: killer in our care homes [online], 2020. Available: https://news.sky.com/video/covid-19-killer-in-our-carehomes-11987237
3 World Health Organization. Preventing and managing covid-19 across long-term care services [online], 2020. Available: https://www. who.int/publications/i/item/WHO-2019-nCoV-Policy_Brief-Longterm_Care-2020.1

4 Comas-Herrera A, Zalakaín J, Lemmon E. Mortality associated with COVID-19 in care homes: international evidence. article in LTCcovid.org, International long-term care policy network, CPECLSE 2020 https://tccovid.org/2020/04/12/mortality-associatedwith-covid-19-outbreaks-in-care-homes-early-internationalevidence/

5 Booth R. Government rejected radical lockdown of England's care homes [online], 2020. Available: https://www.theguardian.com/world/ 2020/may/28/government-rejected-radical-lockdown-england-carehomes-coronavirus

6 Laing W. Impact of coronavirus mortality on care home resident numbers [online], 2020. Available: https://www.laingbuisson.com/wpcontent/uploads/2020/06/Covid-story_v4.pdf

7 Oliver D. Let's not forget care homes when COVID-19 is over. BMJ 2020;369:m1629.

8 Gordon AL, Goodman C, Achterberg W, et al. Commentary: COVID in care homes-challenges and dilemmas in healthcare delivery. Age Ageing 2020;49:701-5.

9 CareEngland. Questions and concerns that have been raised by care England members [online], 2020. Available: http://www.careengland. org.uk/

10 RCN Research Society. Survey of UK nurses and midwives' highlights their concerns about health, training and workload during COVID-19 [online], 2020. Available: https://www.kcl.ac.uk/news/ survey-of-uk-nurses-and-midwives-highlights-their-concerns-abouthealth-training-and-workload-during-covid-19

11 Age UK. Briefing: health and care of older people in England 2019 [online], 2019. Available: https://www.ageuk.org.uk/globalassets/ age-uk/documents/reports-and-publications/reports-and-briefings/ health--wellbeing/age_uk_briefing_state_of_health_and_care_of_ older_people_july2019.pdf

12 Gordon AL, Franklin M, Bradshaw L, et al. Health status of UK care home residents: a cohort study. Age Ageing 2014;43:97-103.

13 British Geriatrics Society. COVID-19: managing the COVID-19 pandemic in care homes for older people [online], 2020. Available: https://www.bgs.org.uk/resources/covid-19-managing-the-covid-19pandemic-in-care-homes

14 GOV.UK. Coronavirus (COVID-19). Admission and care of residents in a care home during COVID-19. Version 2 [online], 2020. Available: https://www.gov.uk/government/publications/coronavirus-covid-19admission-and-care-of-people-in-care-homes

15 Brooks SK, Webster RK, Smith LE, et al. The psychological impact of quarantine and how to reduce it: rapid review of the evidence. Lancet 2020;395:912-20.

16 Suarez-Gonzalez A. Detrimental effects of confinement and isolation in the cognitive and psychological health of people living with dementia during COVID-19: emerging evidence [online], 2020. Available: https://tccovid.org/2020/07/01/detrimental-effects-ofconfinement-and-isolation-on-the-cognitive-and-psychologicalhealth-of-people-living-with-dementia-during-covid-19-emergingevidence/

17 Verbeek H, Gerritsen DL, Backhaus R. Allowing visitors back in the nursing home during the COVID-19 crisis - a Dutch national study into first experiences and impact on well-being. J Am Med Dir Assoc 2020.

18 Billings J, Ching BCF, Gkofa V. Healthcare workers' experiences of working on the frontline and views about support during COVID-19 and comparable pandemics: a rapid review and meta-synthesis. MedRxiv [Preprint] 2020.

19 Wang H, Li T, Barbarino P, et al. Dementia care during COVID-19. Lancet 2020;395:1190-1.

20 Hinsliff-Smith K, Brown J. The experience of family carers and keeping in regular contact with loved ones who permanently live in a care home during the COVID-19 Pandemic: a UK perspective [online], 2020. Available: https://www.dmu.ac.uk/research/centresinstitutes/ihhpsc/project-information.aspx

21 Vadean F, Hussein S, Allan S. Retention and sustainability of social care workforce [online], 2020. Available: https://www.pssru.ac.uk/ resscw/frontpage/

22 Luff R, Laybourne A, Ferreira Z, et al. A guide to research with care homes. Qual Ageing Older Adults 2015;16:186-94.

23 Davies SL, Goodman C, Manthorpe J, et al. Enabling research in care homes: an evaluation of a national network of research ready care homes. BMC Med Res Methodol 2014;14:47.

24 Harris R, Dyson E. Recruitment of frail older people to research: lessons learnt through experience. J Adv Nurs 2001;36:643-51. 
25 Garritty C, Gartlehner G, Nussbaumer-Streit B, et al. Cochrane rapid reviews methods group offers evidence-informed guidance to conduct rapid reviews. J Clin Epidemiol 2021;130:13-22.

26 Stake RE. Qualitative case studies. In: Denzin NK, Lincoln YS, eds. The SAGE handbook of qualitative research. 3rd edn. Thousand Oaks, CA: Sage, 2009: 443-66.

27 Prince M, Knapp M, Guerchet M. Dementia UK: Update alzheimer's society [online], 2014. Available: https://www.dementiastatistics.org/ statistics/care-services/

28 DEEP. Collecting the views of people with dementia, DEEP, 2013. Available: www.dementiavoices.org.uk/wp-content/uploads/2013/ 11DEEP-Guide-Collecting-views.pdf

29 Dementia Enquirers. Dementia enquirers research pack: carrying out your research project. Simple guidance and ideas for DEEP groups. Available: www.dementiavoices.org.uk/wp-content-uploads/2019/06 Dementia-Enquirers-Research-Pack.pdf

30 UK Research Integrity Office. Code of practice for research. Promoting good practice and preventing misconduct [online], 2009. Available: http://ukrio.org/wp-content/uploads/UKRIO-Code-ofPractice-for-Research.pdf
31 Department for Constitutional Affairs. Mental capacity act 2005 code of practice. London: The Stationery Office, 2007. https://assets. publishing.service.gov.uk/government/uploads/system/uploads/ attachment data/file/497253/Mental-capacity-act-code-of-practice.pd

32 Dewing J. Participatory research. A method for process consent with persons who have dementia. Dementia 2007;6:11-25.

33 Samsi K, Manthorpe J. Interviewing people living with dementia in social care. Res Method Rev 2020 https://www.sscr.nihr.ac.uk/wpcontent/uploads/SSCR-methods-review_MR022.pdf

34 Braun C, Clarke C. Successful qualitative research: a practical guide for beginners. London: Sage Publications Ltd, 2014.

35 Miles MB, Huberman M. Qualitative data analysis. Thousand Oaks CA: Sage, 2014

36 Silverman D. Interpreting qualitative data. 5th edn. London: Sage, 2015.

37 Tong A, Sainsbury P, Craig J. Consolidated criteria for reporting qualitative research (COREQ): a 32-item checklist for interviews and focus groups. Int J Qual Health Care 2007;19:349-57. 